\title{
CATÁLOGO FLORÍSTICO DE LA PROVINCIA DE MÁLAGA \\ III. BALANOPHORACEAE - AMARANTHACEAE
}

\section{B. Díez Garretas*}

\section{RESUMEN}

Se continúa la publicación del catálogo florístico de la provincia de Málaga, incluyendo algunas nuevas citas para esta.

\section{SUMMARY}

We continue with a new issue of the floristic catalogue of the Málaga province, in which we include new records.

\section{POLYGONACEAE}

Polygonum equisetiforme Sibth. \& Sm., Fl. Graec. Prodr. 1: 269 (1809). Ruderal.

UF55 Alhaurín de la Torre, 8-IX-1977, Asensi et Diez Garretas (Herb. MÁLAGA 4252). Alhaurín el Grande, 8-IX-1977, Asensi et Diez Garretas (Herb. MÁLAGA 4251).

UF56 Cártama (WILLKomm, 1893:66).

UF76 Málaga (Boissier, 1839-45:552). Ibid. (Ceballos y Vicioso, 1933:166). Ibid., 7-V-1977, Díez Garretas et Alonso (Herb. MALAGA 4250).

Polygonum maritimum L., Sp. Pl. 361 (1753).

Frecuente en todas las playas de la provincia.

UF13 Playa de Cancelada, Estepona, 16-IV-1976, Asensi et Diez Garretas (Herb. MÁLAGA 3717).

UF34 Marbella, 14-IV-1973, Oliva (SEV 14136). Entre Marbella y Elviria, 31-XII-1970, Oliva (SEV 14140 y 14141). Ciudad Sindical de Marbella, 23-XI-1974, Asensi et Díez Garretas (Herb. MÁLAGA 1820).

\footnotetext{
* Departamento de Botánica. Facultad de Ciencias. Universidad de Málaga.
} 
UF54 Playa de la Cala, Mijas, 31-X-1975, Asensi et Díez Garretas (Herb. MÁLAGA 3153).

UF65 Playa de Torremolinos, 16-X-1975, Asensi et Diez Garretas (Herb. MÁLAGA 3150).

UF76 Málaga, VI-1831, Prolongo et Vicioso (MA 20056). Playa de la Misericordia, 30-I-1975, Díez Garretas (Herb. MÁLAGA 1761). Ibid., 4-XII-1974, Asensi et Conde (Herb. MÁLAGA 1772).

VF16 El Morche, 13-IV-1973, Oliva (SEV 14137). Playa entre Lagos y El Morche, 4-VI-1976, Asensi et Díez Garretas (Herb. MÁLAGA 3638).

Polygonum aviculare L., Sp. Pl. 362 (1753).

Muy frecuente como ruderal.

UF06 Ronda (Laza Palacios, 1940:177).

UF34 Marbella (Laza Palacios, 1940:177).

UF65 Los Prados, 29-III-1976, Díez Garretas, Hernández et Asensi (Herb. MÁLAGA 4088).

UF67 Almogía, 27-IV-1974, Antúnez (Herb. MÁLAGA 4108).

UF76 Cerro de San Antón, VII-1934, Laza Palacios (MA 28102). Arroyo de los Pilones, El Palo, 20-IV-1975, Hernández (Herb. MÁLAGA 2439). Cerrado de Calderón, 25-V-1977, Asensi et Diez Garretas (Herb. MÁlAGA 4065). Parque de Málaga, 24-IV-1975, Asensi et Díez Garretas (Herb. MÁLAGA 4106). La Misericordia, 4-V-1975, Asensi et Diez Garretas (Herb. MÁLAGA 4102).

UF86 Playa Cala del Moral, 27-V-1976, Asensi et Diez Garretas (Herb. MÁLAGA 3552).

Polygonum rurivagum Jordan ex Boreau, Fl. Centr. Fr. ed. 3,2:560 (1857).

Polygonum bellardi All.

Raro. Frecuentemente confundido con $P$. aviculare.

UF49 Gobantes, 10-VI-1930, Ceballos et Vicioso (MA 28203).

UF76 Málaga (WILlKomm y LANGE, 1870:289).

UF86 Alrededores del Rincón de la Victoria, 18-VII-1977, Asensi et Díez Garretas (Herb. MÁLAGA 4171).

Polygonum arenastrum Boreau, Fl. Centr. Fr. ed. 3,2:559 (1857).

Al igual que el taxon anterior, suele confundirse con $P$. aviculare.

UF05 Pujerra, 7-X-1977, Asensi et Diez Garretas (Herb. MALAGA 4271).

UF55 Alhaurín el Grande, 8-IX-1977, Asensi et Diez Garretas (Herb. MÁLAGA 4251). 
UF66 Churriana, 30-X-1977, Asensi et Diez Garretas (Herb. MÁLAGA 4294).

UF69 Base del Torcal de Antequera, 1-X-1977, Asensi et Díez Garretas (Herb. MÁLAGA 4243).

UF76 La Misericordia, 13-II-1975, Asensi et Díez Garretas (Herb. MÁLAGA 4107).

UF96 Torre Moya, 16-X-1977, Díez Garretas (Herb. MÁLAGA 4295).

Polygonum salicifolium Brouss. ex Willd., Enum. Pl. Hort. Berol. 1: 428 (1809).

Polygonum serrulatum Lag.

Frecuente en la zona oriental de la provincia, junto a cursos de agua.

UF03 Estepona (WILlKomm y LANGE, $1870: 289$ ).

UF76 Málaga, VI-1915, Gros (MA 28229).

VF06 Torre del Mar, 25-VI-1926, Gros (BC 103826).

VF07 Río de Vélez, 28-VI-1936, Laza Palacios (MAF 851555). Ibid. 5-IX1977, Díez Garretas et Alonso (Herb. MÁLAGA 4176).

Polygonum persicaria L., Sp. Pl. 361 (1753).

Localizada en lugares húmedos.

UF65 La Colina, Torremolinos, 24-III-1975, Asensi et Diez Garretas (Herb. MÁLAGA 4112).

UF76 Vega de Málaga, VI-1972, Laza Rojas (Herb. MÁLAGA 1161).

UF98 Puente de don Manuel, río Alcaucín, 17-X-1975, Hernández et al. (Herb. MÁLAGA 4100).

VF08 Alcaucí n, 17-X-1975, Hernández et al. (Herb. MÁLAGA 4101).

VF26 Vega de Nerja, 3-V-1975, Asensi et Díez Garretas (Herb. MÁlAGA 4113).

Polygonum lapathifolium L., Sp. Pl. 360 (1753).

Frecuente junto a cursos de agua.

UF24 Marbella, desembocadura del río Verde, 19-VII-1973, Cabezudo et Silvestre, (SEV 19664).

UF66 Desembocadura del Guadahlorce, 8-IX-1975, Asensi et Díez Garretas (Herb. MÁLAGA 3069). Ibid., 14-IV-1975, Asensi et Diez Garretas (Herb. MÁLAGA 4111). Ibid., 10-IV-1975, Asensi et Díez Garretas (Herb. MÁlAGA 2340). Ibid., 1-V-1977, Asensi et Díez Garretas (Herb. MALLAGA 4051).

UF76 Málaga (Willkomm y LaNGE, 1870:290).

VF26 Maro, 18-IX-1935, Laza Palacios (MAF 43638). 
Fallopia convolvulus (L.) A. Löve, Taxon, 19:300 (1970).

Bilderdykia convolvulus (L.) Dumort.

Polygonum convolvulus $\mathrm{L}$.

Adoptamos el nombre de Fallopia convolvulus de acuerdo con Galiano y VALDÉs, (1972: 117-142).

UF65 Churriana (Willoomm y LANGE, 1870:291).

VF26 Vega de Nerja, 3-V-1976, Díez Garretas (Herb. MÁLAGA 4280).

Rumex angiocarpus Murb., Lunds Univ. Arsskr. 27(5):46 (1891).

Rumex acetosella L. subsp. angiocarpus Murb.

UF19 Sierra Blanquilla, 19-V-1969, Rivas Goday et Izco (MAF 80356).

UF48 Sierra de Aguas, Carratraca, 7-VI-1965, Rivas Goday (MAF 87409). Ibid. (López GonzÁlez, 1975 : 178).

Rumex acetccella L., Sp. Pl. 338 (1753).

No hemos recolectado esta especie, ni existe testimonio de ella en los herbarios consultados. Las citas de Boissier (1839-45:551) y de Willoomm y LaNGe (1870: $285)$ es probable que correspondan al taxon anterior Rumex angiocarpus $(=R$. acetosella L. subsp. angiocarpus).

Rumex induratus Boiss. \& Reuter, Pugillus 107 (1852).

Muy abundante en toda la provincia, en derrubios y taludes.

UF02 Casares, 15-IV-1975, Asensi et Díez Garretas (Herb. MÁLAGA 3892).

UF03 Sierra Bermeja de Estepona, 16-V-1976, Asensi et Díez Garretas (Herb. MÁlAGA 3493).

UF06 Ronda, 11-VI-1934, Pau (MA 27768).

UF13 Entre Estepona y S. Pedro de Alcántara, 14-V-1971, Cabezudo, Domínguez et Talavera (SEV 9850).

UF26 Yunquera, 21-VII-1975, Asensi (Herb. MÁLAGA 3680). Convento de las Nieves, Sierra de las Nieves, 10-X-1975, Asensi et Hernández (Herb. MÁLAGA 2937).

UF47 Carratraca, 7-V-1973, López González et Valdés Bermejo (MAF 89411).

UF48 Sierra de Aguas, 15-VI-1973, López González et Valdés Bermejo (MAF 89470).

UF54 Faro de Calaburras, Fuengirola, 7-IV-1976, Asensi et Diez Garretas (Herb. MÁlAGA 3354).

UF66 Puerto de la Torre, 27-IV-1975, Asensi et Diez Garretas (Herb. MÁLAGA 4099). 
UF76 Málaga, VI-1915, Gros (MA 27775). Ibid., VIII-1913, Beltrán (MA 27769). Monte Sancha, 29-IV-1977, Asensi et Diez Garretas (Herb. MÁLAGA 4013). Cerro de los Ángeles, 7-III-1935, Laza Palacios (MA 27757). Cerro Coronado, 2-XI-1975, Hernández (Herb. MÁLAGA 3114).

UF86 Rincón de la Victoria, 20-IV-1975, Asensi et Diez Garretas (Herb. MÁLAGA 4094).

UF96 Benagalbón, 8-V-1976, Asensi et Díez Garretas (Herb. MALAGA 4092).

VF06 Torre del Mar, 3-V-1919, Gros (MA 27776). Lagos, 22-I-1975, Díez Garretas (Herb. MÁLAGA 1784).

VF07 Algarrobo, 18-VI-1935, Laza Palacios (MAF 43892).

VF16 Playa del Peñoncillo, Torrox, 10-VI-1976, Asensi et Díez Garretas (Herb. MÁlAGA 3727).

Rumex intermedius DC. in Lam. \& DC., Fl. Fr. ed. 3, 5:369(1815).

UF06 La Nava de S. Luis, 4-VI-1974, Pau et Cuatrecasas, (MAF 43805). Ronda (WILlKomm y LANGE, 1870:186).

UF24 Sierra Blanca, s.f., Silvestre (SEV 7587).

UF45 Alhaurín (WILLKomm y LANGE, $1870: 286$ ).

UF49 Sierra Llana de Antequera, 14-VI-1930, Vicioso (MA 27870).

UF54 Fuengirola, 15-V-1975, Asensi et Diez Garretas (Herb. MÁLAGA 4109).

UF76 Málaga (Willkomm y LaNGe, $1970: 286$ ).

UF77 Pantano del Agujero, 15-IV-1977, Asensi et Diez Garretas (Herb. MÁLAGA 4110).

VF08 Boquete de Zafarraya, 16-VI-1935, Laza Palacios (MAF 43813). Ibid., s.f., Laza Palacios (MAF 85162).

VF17 Cerro Lucero, Frigiliana, VI-1915, Gros (MA 27871).

Rumex crispus L., Sp. Pl. 335 (1753).

Ruderal.

UF76 Málaga, 10-XI-1972, Díez Garretas (Herb. MÁLAGA 691). Delta del Guadalhorce, 10-IX-1977, Díez Garretas (Herb. MÁLAGA 4188).

Rumex conglomeratus Murray, Prodr. Stirp. Gotting. 52 (1770).

Abundante en lugares húmedos.

UF13 Playa de S. Pedro de Alcántara, 19-VI-1976, Asensi et Díez Garretas (Herb. MÁLAGA 3738).

UF26 Sierra de Tolox, 5-VII-1936, Laza Palacios (BCF 5650). 
UF76 La Misericordia, 2-VI-1977, Asensi et Diez Garretas (Herb. MÁLAGA 4145). Ibid., 12-IX-1977, Díez Garretas (Herb. MÁLAGA 4190).

UF89 Villanueva del Trabuco, 16-VI-1976, Hernández et Conde (Herb. MÁLAGA 3587).

VF07 Cercanías de Vélez-Málaga, 2-VI-1977, Díez Garretas (Herb. MÁLAGA 4152).

Rumex pulcher L., Sp. Pl. 336 (1753).

subsp. divaricatus (L.) Murg., Lunds Univ. Arsskr. 27:45 (1891).

Frecuente como ruderal.

TF95 Atajate, 5-VI-1977, Asensi et Diez Garretas (Herb. MÁLAGA 4122). Algatocín, 5-VI-1977, Asensi et Diez Garretas (Herb. MÁLAGA 4121).

UF76 La Misericordia, 15-V-1975, Asensi et Diez Garretas (Herb. MÁLAGA 4104).

UF89 Villanueva del Trabuco, 16-VI-1976, Hernández et al. (Herb. MÁLAGA 3589).

Rumex bucephalophorus L., Sp. Pl. 336 (1753).

subsp. bucephalophorus

Es la subespecie más frecuente en la provincia. Las citas bibliográficas de $R$. bucephalophorus se refieren probablemente a esta subespecie.

TF92 Manilva, 9-V-1932, Vicioso (MA 27560).

UF17 Sierra Blanquilla, Yunquera, 10-VII-1930, Vicioso (MA 27562).

UF26 Tolox, M. Roinvainen, 15-V-1952 (BC 143765). Serranía de Ronda, VI-1930, Ceballos et Vicioso (MA 27557).

UF37 Casarabonela, 9-VI-1973, Asensi (Herb. MALAGA 1163).

UF45 Carretera entre Coín y Mijas, 1-V-1975, Asensi et Diez Garretas (Herb. MÁLAGA 4090).

UF47 Álora, 27-IV-1975, Asensi et Díez Garretas (Herb. MÁLAGA 4105). Ibid., 27-IV-1975, Hernández (Herb. MÁLAGA 2492).

UF48 Sierra de Aguas (López GonzÁlez, 1975:178). Sierra de Carratraca, 20-VI-1930, Vicioso (MA 27558).

UF49 Sierra Llana, 14-VI-1930, Vicioso (MA 27559).

UF54 Faro de Calaburras, Fuengirola, 7-IV-1976, Asensi et Diez Garretas (Herb. MÁlAGA 3385).

UF65 Torremolinos, 1-IV-1935, Laza Palacios (MA 27554).

UF76 Cerrado de Calderón, 20-III-1977, Asensi et Díez Garretas (Herb. MÁlAGA 3924). Monte de las tres letras, 26-II-1973, Asensi et Díez Garretas (Herb. MÁLAGA 690). 
UF86 Rincón de la Victoria, 20-IV-1975, Asensi et Díez Garretas (Herb. MÁLAGA 4098).

VF06 Playa de Benajarafe, 30-V-1976, Asensi et Díez Garretas (Herb. MÁLAGA 3648).

VF08 Sierra Tejeda, 15-V-1977, Asensi et Diez Garretas (Herb. MÁLAGA 4054).

VF16 Torrox (Laza Palacios, 1946:281).

VF26 Arroyo de la Miel, 21-IV-1935, Laza Palacios (MA 27553).

subsp. hispanicus (Steinh.) Rech. fil., Bot. Not. 1939:500 (1939).

Primera cita para la provincia.

UF26 Proximidades de Yunquera, Ronda, 5-VI-1977, Asensi et Diez Garretas (Herb. MÁLAGA 4125).

UF86 Rincón de la Victoria, 8-V-1976, Asensi et Díez Garretas (Herb. MALAGA 4095).

subsp. graecus (Steinh.) Rech. fil. op. cit. 492 (1939).

Únicamente hemos localizado este taxon en las playas de la parte occidental de la provincia. Primera cita para la provincia de Málaga.

TF92 Playa de Chullera, Manilva, 7-IV-1976, Asensi et Diez Garretas (Herb. MÁLAGA 4201).

UF02 Playas del término de Casares, 7-IV-1976, Asensi et Diez Garretas (Herb. MÁLAGA 3440).

UF13 Playa de Cancelada, Estepona, 16-IV-1976, Asensi et Diez Garretas (Herb. MÁlAGA 3798). Playa del Castor, Estepona, 16-IV-1976, Asensi et Diez Garretas (Herb. MÁLAGA 3801).

UF34 Playa de la Ciudad Sindical, Marbella, 22-V-1975, Asensi (Herb. MÁLAGA 2708).

UF44 Playa de Calahonda, Marbella, 20-V-1975, Asensi et al. (Herb. MÁLAGA 2587 y 4103).

subsp. aegaeus Rech. fil., op. cit. 495 (1939).

Escasa. Primera cita para la provincia de Málaga.

UF03 Playa de Chullera, Manilva, 4-VI-1977, Asensi et Díez Garretas (Herb. MÁLAGA 1959).

UF54 Fuengirola, 10-IV-1976, Asensi et Díez Garretas (Herb. MÁLAGA 4093).

Emex spinosa (L.) Campd., Monogr. Rumex 58 (1819).

Muy abundante en toda la provincia como ruderal.

TF92 Playa de Chullera, 7-IV-1976, Asensi et Diez Garretas (Herb. MALAGA 3456). 
UF47 Álora, 27-IV-1975, Asensi et Díez Garretas (Herb. MÁlAGA 4089). Ibid., 27-IV-1975, Hernández (Herb. MÁLAGA 2498).

UF65 Desembocadura del Guadalhorce, 16-IV-1975, Asensi et al. (Herb. MÁLAGA 2449). Campo de Golf, 3-V-1976, Asensi et Hernández (Herb. MÁLAGA 3308).

UF66 Puerto de la Torre, 6-IV-1975, Hernández (Herb. MÁLAGA 2392).

UF67 Cerca de la Venta de las Ánimas, 2-III-1975, Hernández (Herb. MÁLAGA 1952).

UF76 Málaga, 4-II-1976, Asensi et Díez Garretas (Herb. MÁLAGA 3905). Monte de las tres letras, 13-IV-1975, Hernández (Herb. MALAGA 2407). La Misericordia, 19-IV-1975, Hernández (Herb. MÁLAGA 2458).

UF96 Chilches, 4-II-1974, Diez Garretas (Herb. MÁLAGA 453). Benajarafe, 14-II-1976, Asensi et Díez Garretas (Herb. MÁLAGA 4091).

\section{CHENOPODIACEAE}

Beta vulgaris L., Sp. Pl. 222 (1753).

subsp. maritima (L.) Arcángeli, Comp. Fl. Ital. 593 (1882).

Beta maritima L.

Frecuente en lugares próximos al mar.

UF13 Playa de S. Pedro de Alcántara, 19-VI-1976, Asensi et Díez Garretas (Herb. MÁLAGA 3741).

UF65 Playa de Torremolinos, 24-VI-1976, Asensi et Diez Garretas (Herb. MÁLAGA 3756).

UF76 Málaga (Willkomm y Lange, $1870: 274$ ).

UF86 Rincón de la Victoria, 4-VII-1975, Diez Garretas (Herb. MALAGA 2702). Ibid., 15-V-1976, Asensi et Díez Garretas (Herb. MÁLAGA 4097). Playa de la Cala del Moral, 27-V-1976, Asensi et Díez Garretas (Herb. MÁLAGA 3553).

VF06 Playa de Benajarafe, 30-V-1976, Asensi et Díez Garretas (Herb. MÁLAGA 3647).

VF26 Playa de la Torrecilla, Nerja, 4-VI-1976, Asensi et Díez Garretas (Herb. MÁLAGA 3886).

Patellaria cordata J. T. Williams, A. J. Scott \& B. V. Ford-Lloyd, Feddes Repertorium, 87(5):289-292 (1976).

Beta patellaris Moq.

Beta diffusa Cosson.

De acuerdo con J. T. Williams, A. J. Scott y B.V. Ford-Lloyd la sección Patellares del género Beta, debe separarse en un nuevo género, 
Patellaria, correspondiendo Beta patellaris a la nueva denominación de Patellaria cordata.

Poseemos además de la cita indicada, datos de la presencia de este taxon en la provincia de Granada, Playa de los Yesos, 12-VI-1976, Asensi \& Díez Garretas (Herb. MÁlAGA 3650).

UF65 Playa de Torremolinos, 11-IV-54, s. r. (Herb. GDA 4106-b).

Chenopodium botrys L., Sp. Pl. 219 (1753).

VF08 Sierra Tejeda, subida desde Sellera, 850 m, 4-VIII-1977, López González (Herb. MÁLAGA 4317).

Chenopodium ambrosioides L., Sp. Pl. 219 (1753).

Frecuente como ruderal.

UF24 Marbella, Nueva Andalucía, 19-VII-1973, Cabezudo et Silvestre (SEV 16593).

Uf55 La Cala, Mijas, 31-X-1975, Asensi et Díez Garretas (Herb. MALAGA 3158). Alhaurín el Grande, 8-IX-1977, Asensi et Diez Garretas (Herb. MALAGA 4221).

UF65 Desembocadura del Guadalhorce, 5-IX-1975, Asensi et Diez Garretas (Herb. MÁLAGA 4286). Ibid., 9-IX-1975, Díez Garretas (Herb. MALAGA 3068).

UF76 La Misericordia, 8-X-1975, Hernández et Diez Garretas (Herb. MALAGA 2991). Málaga (WillKomm y LANGE, 1870:271).

UF86 Rincón de la Victoria, 18-VII-1977, Asensi et Díez Garretas (Herb. MÁLAGA 4163).

VF07 Vélez (Willkomm y Lange, 1870:271). Vélez-Málaga, carretera a Colmenar, 13-VII-1977, Díez Garretas (Herb. MÁLAGA 4154).

Chenopodium glaucum L., Sp. Pl. 220 (1753).

Citada por Boissier, pero no encontrada por nosotros.

UF06 Ronda (BOISSIER, 1839-45:540).

UF76 Málaga (BoIssier, 1839-45:540).

Chenopodium vulvaria L., Sp. Pl. 220 (1753).

Escasa.

UF69 Base del Torcal de Antequera, junto a la Venta de la Sierra, 1-X1977, Asensi et Diez Garretas (Herb. MALAGA 4244).

Chenopodium murale L., Sp. Pl. 219 (1753).

Muy común.

UF37 Carratraca (Willkomm y Lange, 1870:273). 
UF47 Álora, 12-IX-1977, Asensi et Díez Garretas (Herb. MÁLAGA 4187 y 4192).

UF69 Base del Torcal de Antequera, 2-X-1977, Asensi et Díez Garretas (Herb. MÁLAGA 4245).

UF76 Málaga (Willkomm y LaNGe, 1870:273). Ibid., 4-XI-1976, Asensi et Diez Garretas (Herb. MÁLAGA 3897). Cerrado de Calderón, 15-VII-1977, Diez Garretas (Herb. MÁLAGA 4169).

UF78 Casabermeja, 12-III-1972, Laza Rojas (Herb. MALAGA 1152).

UF86 Playa de la Cala del Moral, 27-V-1976, Asensi et Diez Garretas (Herb. MÁlAGA 3548). Alrededores del Rincón de la Victoria, 18-VII-1977, Asensi et Diez Garretas (Herb. MÁLAGA 4164).

UF96 Playa de Benajarafe, 30-V-1976, Asensi et Díez Garretas (Herb. MÁLAGA 3649 y 3887).

VF07 Vélez-Málaga, carretera a Colmenar, 13-VII-1977, Díez Garretas (Herb. MÁLAGA 4158).

VF08 Sierra Tejeda, 15-V-1977, Asensi et Díez Garretas (Herb. MÁLAGA 4045).

VF26 Nerja, 10-II-1973, Asensi et Díez Garretas (Herb. MALAGA 297).

Chenopodium opulifolium Schrader ex Koch \& Ziz, Fl. Palat. 6 (1814). Muy frecuente como ruderal.

UF55 Alhaurín el Grande, 8-IX-1977, Asensi et Díez Garretas (Herb. MÁLAGA 4219). Alhaurín de la Torre, 8-IX-1977, Asensi et Diez Garretas (Herb. MALAGA 4216).

UF65 Playa de Torremolinos, 16-X-1975, Asensi et Díez Garretas (Herb. MÁLAGA 4284).

UF76 La Misericordia, 14-V-1975, Asensi et Diez Garretas (Herb. MALAGA 4282). Málaga (Willkomm y LaNGe, 1870:272).

UF86 Alrededores del Rincón de la Victoria, 19-VII-1977, Díez Garretas (Herb. MALAGA 4162).

UF96 Benajarafe, 18-VII-1977, Diez Garretas (Herb. MÁLAGA 4161).

Chenopodium album L., Sp. Pl. 219 (1753).

Muy frecuente en toda la provincia.

UF24 Marbella, Nueva Andaiucía, 19-VII-1973, Cabezudo et Silvestre (SEV 16594).

UF26 Convento de las Nieves, Sierra de las Nieves, 10-X-1975, Asensi et Hernández (Herb. MÁLAGA 4285).

UF55 La Cala, Mijas 31-X-1975, Asensi et Diez Garretas (Herb. MÁLAGA 3160). Alhaurín de la Torre, 8-IX-1977, Asensi et Díez Garretas (Herb. MÁLAGA 4218). 
UF56 Aljaima, 12-IX-1977, Asensi et Díez Garretas (Herb. MÁLAGA 4196).

UF65 Playa de Torremolinos, 16-X-1975, Asensi et Diez Garretas (Herb. MÁLAGA 3143 y 3147).

UF76 La Misericordia, 8-IX-1975, Hernández et Díez Garretas (Herb. MÁLAGA 2992). Málaga, 10-IX-1977, Asensi et Díez Garretas (Herb. MÁlAGA 4194). Málaga, finca "La Cerda", XI-1914, s.r. (MAF 45895).

UF96 Playa de Benajarafe, 30-V-1976, Asensi et Diez Garretas (Herb. MÁLAGA 3884).

UG70 Archidona, 15-X-1975, Hernández (Herb. MÁLAGA 3131).

Atriplex halimus L., Sp. Pl. 1052 (1753).

En lugares próximos al mar.

UF65 Playa de Torremolinos, 16-X-1975, Asensi et Diez Garretas (Herb. MÁLAGA 3144). Ibid., 24-VI-1976, Asensi et Diez Garretas (Herb. MÁLAGA 3729).

VF26 Nerja, 12-VI-1976, Asensi et Diez Garretas (Herb. MÁLAGA 3674). Ibid., (Laza Palacios, 1946:282).

Atriplex rosea L., Sp. Pl. ed. 2, 1493 (1763).

Aunque Willкomm la cita de Málaga en su Prodromus, dudamos de su existencia en la provincia.

Atriplex littoralis L., Sp. Pl. 1054 (1753).

Poco frecuente.

UF26 Convento de las Nieves, Sierra de las Nieves, 10-X-1975, Asensi et Hernández (Herb. MÁLAGA 4227).

UF76 Málaga, VIII, Rivas Mateos (MAF 67422).

Atriplex patula L., Sp. Pl. 1053 (1753).

Poco frecuente.

UG41 Laguna de Fuente Piedra, 1-X-1977, Asensi et Díez Garretas (Herb. MÁLAGA 4241).

Atriplex hastata L., Sp. Pl. 1053 (1753).

Frecuente como ruderal.

UF34 Chapas de Marbella, 6-IX-1977, Asensi et Diez Garretas (Herb. MÁLAGA 4180).

UF48 Sierra de Aguas, Carratraca, 10-IX-1972, López González (MAF 89343). 
UF55 La Cala, Mijas, 31-X-1975, Asensi et Diez Garretas (Herb. MÁLAGA 3157).

UF65 Playa del Campo de Golf, 16-X-1975, Asensi et Diez Garretas (Herb. MÁLAGA 3149). Desembocadura del Guadalhorce, 10-X1975, Asensi et Díez Garretas (Herb. MÁLAGA 3077). Ibid., 16-IV1975, Asensi et al. (Herb. MÁLAGA 2430).

UF76 La Misericordia, 19-VI-1975, Hernández (Herb. MÁLAGA 2478).

Halimione portulacoides (L.) Aellen, Verhandl. Naturf. Gesell. Basel, 49 : 126 (1938).

Atriplex portulacoides L.

Obione portulacoides (L.) Moq.

En lugares salinos.

UF65 Desembocadura del Guadalhorce, 10-IX-1975, Diez Garretas (Herb. MÁlAGA 3074). Ibid., 24-VI-1976, Asensi et Díez Garretas (Herb. MÁLAGA 3734).

UF76 Playa de la Misericordia, 4-XII-1974, Díez Garretas et Conde (Herb. MÁlAGA 1766). Ibid., 30-I-1975, Diez Garretas et Conde (Herb. MÁLAGA 1856).

Bassia hyssopifolia (Pallas) Volk. in Engler \& Prantl, Natürl. Pflanzenfam. 3 (1a): 70 (1893).

Kochia hyssopifolia (Pallas) Schrader

En lugares salinos, solamente la hemos encontrado en el delta del Guadalhorce. No citada hasta ahora para la provincia.

UF65 Desembocadura del Guadalhorce, 10-IX-1975, Diez Garretas (Herb. MÁLAGA 3075).

Halopeplis amplexicaulis (Vahl) Ung.-Sternb. ex Cesati, Passer. \& Gibelli, Comp. Fl. Ital. 271 (1869).

Halostachys perfoliata sensu Willk.

La única localidad de esta especie en la provincia, es la laguna salada de Fuente Piedra. Actualmente está restringida a una pequeña población. La cita de Rivas GodAy de Antequera se refiere con toda seguridad a dicha laguna.

UG41 Fuente Piedra, 30-IX-1932, Vicioso (MA 29363). Ibid., 2-X-1977, Asensi et Diez Garretas (Herb. MÁLAGA 4249). Antequera, 24-VII1961, Rivas Goday (MAF 90933 y 90934). 
Arthrocnemum fruticosum (L.) Moq., Chenop. Enum. 111 (1840).

Salicornia fruticosa (L.) L.

Escasa. Muy localizada.

UF76 Playa de la Misericordia, 4-XII-1974, Asensi et Díez Garretas (Herb. MÁlAGA 1760). Málaga (Willkomm, 1893:61).

Arthrocnemum glaucum (Delile) Ung.-Sternb., Atti Congr. Bot. Firenze 283 (1876).

Salicornia macrostachya Moric.

Las únicas poblaciones de este taxon, al igual que Halopeplis amplexicaulis, están en la laguna salada de Fuente Piedra. La cita de Rivas GodaY de Antequera se refiere con toda seguridad a dicha laguna.

UG41 Fuente Piedra, 3-IX-1932, Vicioso (MA 29385). Ibid., 6-III-1976, Asensi et Díez Garretas (Herb. MÁLAGA 3236). Antequera, 22-VI1961, Rivas Goday (MAF 90928).

Suaeda vera J. F. Gmelin in L., Syst. Nat. ed. 13, $2(1$ : 503 (1791).

Sueda fruticosa auct.

Muy localizada al igual que el taxon anterior.

UF41 Fuente Piedra, 30-IX-1932, Vicioso (MA 29554). Ibid., 6-III-1976, Asensi et Diez Garretas (Herb. MÁLAGA 3195). Ibid., 21-VI-1977, Asensi et Díez Garretas (Herb. MÁLAGA 4129).

Suaeda maritima (L.) Dumort., Fl. Belg. 22 (1827).

subsp. maritima

Escasa.

UF65 Desembocadura del Guadalhorce, 10-IX-1975, Diez Garretas (Herb. MÁLAGA 3072).

Suaeda splendens (Pourret) Gren \& Godron, Fl. Fr. $3: 30$ (1855).

Las únicas citas corresponden a la laguna de Fuente Piedra.

UG41 Fuente Piedra, 30-IX-1932, Vicioso (BC 96973). Ibid., 30-IX-1932, Vicioso (MA 29488). Ibid., 13-VII-1977, Díez Garretas (Herb. MÁLAGA 4159).

Salsola kali L., Sp. Pl. 222 (1753).

subsp. kali

Frecuente a lo largo de las playas, aunque a veces también se encuentra en el interior como ruderal.

UF34 Playa de la Ciudad Sindical de Marbella, 11-XI-1974, Asensi (Herb. MÁLAGA 1796). 
UF44 Sitio de Calahonda, Marbella, 1-VII-1975, Díez Garretas (Herb. MÁLAGA 4229).

UF55 Alhaurín de la Torre, 8-IX-1977, Asensi et Díez Garretas (Herb. MÁLAGA 4217).

UF65 Desembocadura del Guadalhorce, 10-IX-1975, Asensi et Díez Garretas (Herb. MÁLAGA 3142).

UF76 Málaga, 12-V-1972, Laza Rojas (Herb. MÁLAGA 298).

VF06 Torre del Mar (LAZA Palacios, 1946:282).

VF26 Playa de Maro, 3-X-1975, Asensi et Diez Garretas (Herb. MÁLAGA 2986).

Salsola verticillata Schousboe, Vextr. Marokko 123 (1800).

Salsola longifolia sensu Willk.

Escasa. Muy localizada.

UF76 Málaga (Ceballos y Vicioso, 1933:167). Málaga, V-1972, Laza Rojas (Herb. MALAGA 292).

Salsola vermiculata L., Sp. Pl. 323 (1753).

Ceballos y Vicioso (1933:167), la incluyen en su catálogo de plantas leñosas basándose en esta misma cita de Reverchon.

UF06 Ronda, 17-VIII-1889, Reverchon (MA 29822).

\section{AMARANTHACEAE}

Amaranthus hybridus L. Sp. Pl. 990 (1753).

Amaranthus chlorostachys Willd.

Muy abundante como ruderal.

UF05 Pujerra, 7-X-1977, Asensi et Diez Garretas (Herb. MALAGA 4293).

UF47 Alora, 12-IX-1977, Asensi et Díez Garretas (Herb. MÁLAGA 4193).

UF55 Alhaurín el Grande, 8-IX-1977, Asensi et Diez Garretas (Herb. MÁLAGA 4223 y 4272).

UF56 Cártama, 13-VIII-1888, Reverchon (MA 29962).

UF69 Base del Torcal de Antequera, Venta de la Sierra, 2-X-1977, Asensi et Díez Garretas (Herb. MÁLAGA 4246).

UF76 La Misericordia, 8-X-1975, Hernández et Diez Garretas (Herb. MALAGA 2988). Málaga, 9-IX-1977, Asensi et Diez Garretas (Herb. MÁLAGA 4225).

UF86 Alrededores del Rincón de la Victoria, 18-VII-1977, Asensi et Diez Garretas (Herb. MÁLAGA 4165). 
UF96 Torre Moya, 16-X-1977, Diez Garretas (Herb. MÁLAGA 4297).

VF07 Cercanías de Vélez-Málaga, 13-VII-1977, Díez Garretas (Herb. MÁLAGA 4155).

Amaranthus cruentus L., Syst. Nat. ed. 10, 2:1269 (1759).

Amaranthus patulus Bertol.

Poco frecuente.

UF46 Sierra Gorda, Coín, 31-VIII-1977, Diez Garretas et Alonso (Herb. MÁLAGA 4177).

UF76 La Misericordia, 26-X-1977, Díez Garretas (Herb. MÁLAGA 4299).

Amaranthus paniculatus L., Sp. Pl. ed. 2, 1406 (1763).

UG41 Fuente Piedra, 10-XI-1977, Asensi et Diez Garretas (Herb. MÁLAGA 4303).

Amaranthus retroflexus L., Sp. $P l .991$ (1753).

Poco frecuente.

UF48 Sierra de Aguas, Carratraca (LóPEZ GonZÁLEZ, 1975:116).

UF76 La Misericordia, 26-X-1977, Díez Garretas (Herb. MALAGA 4298).

Amaranthus muricatus (Moq.) Gillies ex Hicken, Apunt. Hist. Nat.

(Buenos Aires) 2:92 (1910).

Relativamente frecuente en la provincia.

UF47 Álora, 12-IX-1977, Díez Garretas (Herb. MÁLAGA 4195).

UF76 La Misericordia, 8-X-1975, Hernández et Díez Garretas (Herb. MÁLAGA 2990). Ibid., 12-VII-1977, Díez Garretas (Herb. MALAGA 4166).

Amaranthus blitoides S. Watson, Proc. Amer. Acad. Arts Sci. 12:273 (1877).

Muy frecuente como ruderal en toda la provincia.

UF24 Nueva Andalucía, Marbella, desembocadura del río Verde, 19-VII1973, Cabezudo et Silvestre (SEV 19643).

UF35 Monda, 29-X-1977, Asensi et Diez Garretas (Herb. MÁLAGA 4304).

UF47 Álora, 12-IX-1977, Díez Garretas (Herb. MÁLAGA 4185). Sierra Gorda, Coín, 31-VII-1977, Alonso (Herb. MÁLAGA 4175).

UF54 Desembocadura del río Fuengirola, 16-VII-1975, Díez Garretas (Herb. MÁlAGA 2724). La Cala, Mijas, 31-X-1975, Asensi et Díez Garretas (Herb. MÁLAGA 3159). 
UF55 Alhaurín de la Torre, 8-IX-1977, Asensi et Diez Garretas (Herb. MÁLAGA 4181). Alhaurín el Grande, 8-IX-1977, Asensi et Diez Garretas (Herb. MÁLAGA 4220).

UF65 Desembocadura del Guadalhorce, 8-IX-1975, Diez Garretas (Herb. MÁLAGA 3066). Ibid., 10-IV-1975, Hernández et Díez Garretas (Herb. MÁLAGA 2339). Playa de Torremolinos, 16-X-1975, Asensi et Díez Garretas (Herb. MÁLAGA 3146).

UF76 La Misericordia, 8-X-1975, Hernández et Diez Garretas (Herb. MÁLAGA 2993). Cerrado de Calderón, 25-V-1977, Asensi et Díez Garretas (Herb. MÁLAGA 4064).

UF86 Alrededores del Rincón de la Victoria, 18-VII-1977, Díez Garretas (Herb. MÁLAGA 4172).

VF07 Cercanías de Vélez-Málaga, 13-VII-1977, Diez Garretas (Herb. MÁLAGA 4153).

Amaranthus albus L., Syst. Nat. ed. 10, 2:1268 (1759).

UF05 Pujerra, 7-X-1977, Asensi et Díez Garretas (Herb. MÁLAGA 4257).

UF06 Ronda, IX-1931, Ceballos et Vicioso (MA 29991 (2).

UF47 Alora, 12-IX-1977, Díez Garretas (Herb. MALAGA 4183).

UF48 Sierra de Aguas (López GonzÁlez, 1975: 115).

UF55 Alhaurín de la Torre, 8-IX-1977, Diez Garretas (Herb. MÁLAGA 4182).

Amaranthus deflexus L., Mantissa Altera 295 (1771).

UF48 Sierra de Aguas (López GonzÁlez, 1975: 115).

UF55 Alhaurín el Grande, 8-IX-1977, Asensi et Diez Garretas (Herb. MÁLAGA 4222).

UF98 Puente de don Manuel, 13-VII-1977, Díez Garretas (Herb. MÁLAGA 4156).

Amaranthus lividus L., Sp. Pl. 990 (1753).

Amaranthus blitum L.

Muy abundante en toda la provincia.

UF35 Monda, 29-X-1977, Asensi et Díez Garretas (Herb. MÁlAGA 4305).

UF38 Carratraca, 12-IX-1977, Asensi et Diez Garretas (Herb. MÁLAGA 4197).

UF47 Álora, 12-IX-1977, Asensi et Diez Garretas (Herb. MÁLAGA 4191).

UF54 La Cala, Mijas, 31-X-1975, Asensi et Diez Garretas (Herb. MALAGA 3161).

UF55 Alhaurín de la Torre, 8-IX-1977, Asensi et Diez Garretas (Herb. MÁLAGA 4226). 
UF76 La Misericordia, 8-X-1975, Hernández et Díez Garretas (Herb. MÁlAGA 2989). Málaga, 8-VI-1977, Asensi et Díez Garretas (Herb. MÁLAGA 4126). Cerrado de Calderón, 15-VII-1977, Diez Garretas (Herb. MÁLAGA 4168).

UF86 Alrededores Rincón de la Victoria, Asensi et Díez Garretas (Herb. MÁLAGA 4170).

VF07 Cercanías de Vélez-Málaga, 13-VII-1977, Asensi et Díez Garretas (Herb. MÁLAGA 4160).

\section{BIBLIOGRAFIA}

Boissier, E. 1839-45. Voyage botanique dans le midi de l'Espagne. Vol. 2. París. Ceballos, L. \& Vicioso, C. 1933. Estudio sobre la vegetación y flora de la provincia de Málaga. Inst. For. Inv. Exp. Madrid.

Galiano, E. F. \& Valdés, B. 1972. Catálogo de las plantas vasculares de la provincia de Sevilla. II. Pinaceae-Polygonaceae. Lagascalia, 2(1):117-142. Sevilla.

Laza Palacios, M. 1940. Flórula farmacéutica malacitana. Anal. R. Acad. Farm., 6:165-214. Madrid.

Laza Palacios, M. 1946. Estudios sobre la flora y la vegetación de las sierras Tejeda y Almijara. Anal. Jard. Bot. Madrid, 6(2):217-370. Madrid.

López González, G. 1975. Contribución al estudio florístico y fitosociológico de Siera de Aguas. Act. Bot. Malacitana, 1:81-205. Málaga.

Maire, R. 1961-1962. Flore de l'Afrique du Nord. Vol. 7 y 8. Ed. Paul Lechevalier. París.

Tutin, T. G., Heywood, V. H. \& col, 1964. Flora Europaea, 1. Cambridge.

Williams, J. T., Scott, A. J. \& Ford-Lloyd, B. V. 1976. Patellaria: a new genus in the Chenopodiaceae. Feddes Repertorium, 87(5):289-292. Berlín.

Willкомм, M. 1893. Supplementum Prodromi Florae Hispanicae. Stuttgart.

Willkomm, M. \& Lange, J. 1870. Prodromus Florae Hispanicae, 1. Stuttgart. 